\title{
Well-posedness and exponential stability of a thermoelastic-Bresse system with second sound and delay
}

\author{
Gang Li(D), Yue Luan (D), Wenjun Liu*(i) \\ College of Mathematics and Statistics, Nanjing University of Information Science and Technology, \\ Nanjing 210044, China
}

\begin{abstract}
In this paper, we consider a one-dimensional thermoelastic-Bresse system with a delay term, where the heat conduction is given by Cattaneo's law effective in the shear angle displacement. We prove that the system is well-posed by using the semigroup method, and show, using the multiplier method, that the dissipation induced by the heat is strong enough to exponentially stabilize the system in the presence of a "small" delay when the stable number is zero.
\end{abstract}

Mathematics Subject Classification (2010). 35L53, 35L05, 93C20, 93D20

Keywords. thermoelastic-Bresse system, second sound, exponential decay, time delay

\section{Introduction}

In this paper, we consider the following thermoelastic-Bresse system with a constant internal delay:

$$
\begin{cases}\rho_{1} \varphi_{t t}-k\left(\psi+\varphi_{x}+l \omega\right)_{x}-l k_{0}\left(\omega_{x}-l \varphi\right)+\mu \varphi_{t}\left(x, t-\tau_{0}\right)=0, & (x, t) \in(0,1) \times(0,+\infty), \\ \rho_{2} \psi_{t t}-b \psi_{x x}+k\left(\varphi_{x}+\psi+l \omega\right)+\gamma \theta_{x}=0, & (x, t) \in(0,1) \times(0,+\infty), \\ \rho_{1} \omega_{t t}-k_{0}\left(\omega_{x}-l \varphi\right)_{x}+l k\left(\varphi_{x}+\psi+l \omega\right)=0, & (x, t) \in(0,1) \times(0,+\infty), \\ \rho_{3} \theta_{t}+q_{x}+\gamma \psi_{t x}=0, & (x, t) \in(0,1) \times(0,+\infty), \\ \tau q_{t}+\beta q+\theta_{x}=0, & (x, t) \in(0,1) \times(0,+\infty), \\ \varphi(x, 0)=\varphi_{0}(x), \varphi_{t}(x, 0)=\varphi_{1}(x), \theta(x, 0)=\theta_{0}(x), & x \in[0,1], \\ \psi(x, 0)=\psi_{0}(x), \psi_{t}(x, 0)=\psi_{1}(x), q(x, 0)=q_{0}(x), & x \in[0,1], \\ \omega(x, 0)=\omega_{0}(x), \omega_{t}(x, 0)=\omega_{1}(x), \varphi_{t}(x,-t)=f_{0}(x, t), & x \in[0,1], t \in(0, \tau), \\ \varphi(0, t)=\varphi_{x}(1, t)=\psi_{x}(0, t)=\psi(1, t)=0, & t \in[0,+\infty), \\ \omega_{x}(0, t)=\omega(1, t)=\theta(0, t)=q(1, t)=0, & t \in[0,+\infty) .\end{cases}
$$

*Corresponding Author.

Email addresses: ligang@nuist.edu.cn (G. Li), yluan_sunflower@163.com (Y. Luan), wjliu@nuist.edu.cn (W. Liu)

Received: 23.09.2017; Accepted: 11.01.2019 
This is a thermoelastic system of Bresse type $([3,12,13])$ which governs the mechanical deformations in elastic structures of circular arch type, where the heat flux is given by Cattaneo's law. It is composed of five functions, three of which representing the mechanical deformations: the longitudinal displacement $\omega$, the vertical displacement $\varphi$ and the shear angle displacement $\psi ; \theta$ is the difference temperature, $q$ is the heat flux $([15,20,21])$. The coefficients $\rho_{i}(i=1,2,3), k, l, k_{0}, b, k, \gamma, \tau, \beta$ are positive constants, $\mu$ is a real number, and $\tau_{0}>0$ represents the time delay.

With respect to asymptotic behavior of solutions for thermoelastic Bresse systems, some results can be obtained. Fatori and Rivera [8] considered Bresse system with thermal dissipation effective only in one equation wrote as

$$
\begin{cases}\rho_{1} \varphi_{t t}-k\left(\psi+\varphi_{x}+l \omega\right)_{x}-l k_{0}\left(\omega_{x}-l \varphi\right)=0, & (x, t) \in(0,1) \times(0,+\infty), \\ \rho_{2} \psi_{t t}-b \psi_{x x}+k\left(\varphi_{x}+\psi+l \omega\right)+\gamma \theta_{x}=0, & (x, t) \in(0,1) \times(0,+\infty), \\ \rho_{1} \omega_{t t}-k_{0}\left(\omega_{x}-l \varphi\right)_{x}+l k\left(\varphi_{x}+\psi+l \omega\right)=0, & (x, t) \in(0,1) \times(0,+\infty), \\ \theta_{t}-k_{1} \theta_{x x}+m \psi_{x t}=0, & (x, t) \in(0,1) \times(0,+\infty),\end{cases}
$$

and showed that there exist exponential stability if and only if the wave propagation is equal. They also showed that, in general, the system is not exponentially stable but that there exists polynomial stability with rates that depend on the wave propagations and the regularity of the initial data. In [10], Keddi et al. studied the well-posedness and the asymptotic stability of a one-dimensional thermoelastic Bresse system, where the heat conduction is given by Cattaneo's law effective in the shear angle displacement, wrote as

$$
\begin{cases}\rho_{1} \varphi_{t t}-k\left(\psi+\varphi_{x}+l \omega\right)_{x}-l k_{0}\left(\omega_{x}-l \varphi\right)=0, & (x, t) \in(0,1) \times(0,+\infty), \\ \rho_{2} \psi_{t t}-b \psi_{x x}+k\left(\varphi_{x}+\psi+l \omega\right)+\gamma \theta_{x}=0, & (x, t) \in(0,1) \times(0,+\infty), \\ \rho_{1} \omega_{t t}-k_{0}\left(\omega_{x}-l \varphi\right)_{x}+l k\left(\varphi_{x}+\psi+l \omega\right)=0, & (x, t) \in(0,1) \times(0,+\infty), \\ \rho_{3} \theta_{t}+q_{x}+\gamma \psi_{t x}=0, & (x, t) \in(0,1) \times(0,+\infty), \\ \tau q_{t}+\beta q+\theta_{x}=0, & (x, t) \in(0,1) \times(0,+\infty) .\end{cases}
$$

They established the well-posedness of the system and proved that the system was exponentially stable depending on the stable number of the system, and showed that in general, the system was polynomially stable. If $l \equiv 0$, Bresse system reduces to the well-known Timoshenko system (see $[1,5-7,14,22])$.

Time delays so often arise in many physical, chemical, biological, thermal and economical phenomena (see $[4,9,16-19,23-25,27,29-34]$ ). The presence of delay may be a source of instability. In recent years, the control of partial differential equations with time delay effects has become an active area of research. For example, Kafini et al [9] studied the Timoshenko system of thermoelasticity of type III with delay of the form

$$
\begin{cases}\rho_{1} \phi_{t t}-k\left(\phi_{x}+\psi\right)_{x}+\mu_{1} \phi_{t}(x, t)+\mu_{2} \phi_{t}(x, t-\tau)=0, & (x, t) \in(0,1) \times(0,+\infty), \\ \rho_{2} \psi_{t t}-b \psi_{x x}+k\left(\phi_{x}+\psi\right)+\beta \theta_{t x}=0, & (x, t) \in(0,1) \times(0,+\infty), \\ \rho_{3} \theta_{t t}-\delta \theta_{x x}+\gamma \psi_{t x}-K \theta_{t x x}=0, & (x, t) \in(0,1), \times(0,+\infty), \\ \theta(\cdot, 0)=\theta_{0}, \theta_{t}(\cdot, 0)=\theta_{1}, \psi(\cdot, 0)=\psi_{0}, & x \in[0,1], \\ \psi_{t}(\cdot, 0)=\psi_{1}, \phi(\cdot, 0)=\phi_{0}, \phi_{t}(\cdot, 0)=\phi_{1}, & x \in[0,1], \\ \phi_{t}(x, t-\tau)=f_{0}(x, t-\tau), & t \in[0, \tau], \\ \phi(0, t)=\phi(1, t)=\psi(0, t)=\psi(1, t)=\theta_{x}(0, t)=\theta_{x}(1, t)=0 . & t \in[0,+\infty),\end{cases}
$$

and proved that under suitable conditions on the initial data the energy decays exponentially in the case of equal wave speeds in spite of the existence of the delay. And they also got the result that the energy decays polynomially under different wave speeds 
assumption. In [2], Apalara and Messaoudi considered the following one-dimensional linear thermoelastic system of Timoshenko type with delay, where the heat flux is given by Cattaneo's law:

$$
\left\{\begin{array}{l}
\rho_{1} \varphi_{t t}-k\left(\psi+\varphi_{x}\right)_{x}+\mu \varphi_{t}\left(x, t-\tau_{0}\right)=0, \quad(x, t) \in(0,1) \times(0,+\infty), \\
\rho_{2} \psi_{t t}-b \psi_{x x}+k\left(\varphi_{x}+\psi\right)+\gamma \theta_{x}=0, \quad(x, t) \in(0,1) \times(0,+\infty), \\
\rho_{3} \theta_{t}+q_{x}+\gamma \psi_{t x}=0, \quad(x, t) \in(0,1) \times(0,+\infty), \\
\tau q_{t}+\beta q+\theta_{x}=0, \quad(x, t) \in(0,1) \times(0,+\infty), \\
\varphi(x, 0)=\varphi_{0}(x), \varphi_{t}(x, 0)=\varphi_{1}(x), \psi(x, 0)=\psi_{0}(x), \psi_{t}(x, 0)=\psi_{1}(x), \quad x \in[0,1], \\
\theta(x, 0)=\theta_{0}(x), q(x, 0)=q_{0}(x), \varphi_{t}(x,-t)=f_{0}(x, t), \quad x \in[0,1], \\
\varphi(0, t)=\varphi(1, t)=\psi_{x}(0, t)=\psi_{x}(1, t)=\theta(0, t)=\theta(1, t)=0, \quad t \in(0,+\infty) .
\end{array}\right.
$$

They proved an exponential decay result under a smallness condition on the delay and a stability number, and reproduced the polynomial decay of Santos et al. [28] using the multiplier method in the case of absence of delay.

Based on the above results, in this paper, we study the thermoclastic-Bresse system (1.1) with second sound and delay. Introducing a delay term in the internal feedback of thermoclastic-Bresse system with second sound makes our problem different from those considered so far in the literature (such as [10]). For our purpose, we use the idea of Apalara and Messaoudi in [2] to take into account the effect of the delay. We first use the semigroup method to prove the well-posedness result of the system. Then, we show, using the multiplier method, that the dissipation induced by the heat is strong enough to stabilize the system in the presence of a "small" delay when the stable number is zero.

The remaining part of this paper is organized as follows. In Section 2, we establish the well-posedness result of the system. In Section 3, we give the exponential decay result by modifying some classical multipliers.

\section{Well-posedness}

In this section, we use the semigroup techniques to prove the well-posedness of problem (1.1). In order to exhibit the dissipative nature of system (1.1), as in [23], we introduce the new variable

$$
z(x, \rho, t)=\varphi_{t}\left(x, t-\rho \tau_{0}\right) \quad x \in(0,1), \rho \in(0,1), t>0 .
$$

A simple differentiation shows that the variable satisfies

$$
\tau_{0} z_{t}(x, \rho, t)+z_{\rho}(x, \rho, t)=0 \quad x \in(0,1), \rho \in(0,1), t>0 .
$$


Hence, problem (1.1) is equivalent to the following:

$$
\begin{cases}\rho_{1} \varphi_{t t}-k\left(\psi+\varphi_{x}+l \omega\right)_{x}-l k_{0}\left(\omega_{x}-l \varphi\right)+\mu z(x, 1, t)=0, & (x, t) \in(0,1) \times(0,+\infty), \\ \rho_{2} \psi_{t t}-b \psi_{x x}+k\left(\varphi_{x}+\psi+l \omega\right)+\gamma \theta_{x}=0, & (x, t) \in(0,1) \times(0,+\infty), \\ \rho_{1} \omega_{t t}-k_{0}\left(\omega_{x}-l \varphi\right)_{x}+l k\left(\varphi_{x}+\psi+l \omega\right)=0, & (x, t) \in(0,1) \times(0,+\infty), \\ \rho_{3} \theta_{t}+q_{x}+\gamma \psi_{t x}=0, & (x, t) \in(0,1) \times(0,+\infty), \\ \tau q_{t}+\beta q+\theta_{x}=0, & (x, t) \in(0,1) \times(0,+\infty), \\ \tau_{0} z_{t}(x, \rho, t)+z_{\rho}(x, \rho, t)=0, & (x, t) \in(0,1) \times(0,+\infty), \\ \varphi(x, 0)=\varphi_{0}(x), \varphi_{t}(x,-t)=f_{0}(x, t), \theta(x, 0)=\theta_{0}(x), & x \in[0,1], \\ \psi(x, 0)=\psi_{0}(x), \psi_{t}(x, 0)=\psi_{1}(x), q(x, 0)=q_{0}(x), & x \in[0,1], t \in(0, \tau), \\ \omega(x, 0)=\omega_{0}(x), \omega_{t}(x, 0)=\omega_{1}(x), z(x, 0, t)=\varphi_{t}(x, t), & x \in[0,1], t \in(0,+\infty), \\ \varphi(0, t)=\varphi_{x}(1, t)=\psi_{x}(0, t)=\psi(1, t)=0, & t \in[0,+\infty), \\ \omega_{x}(0, t)=\omega(1, t)=\theta(0, t)=q(1, t)=0, & t \in[0,+\infty) .\end{cases}
$$

Now, we let

$$
\Phi=(\varphi, u, \psi, v, \omega, w, \theta, q, z),
$$

then system (2.1) can be written as an evolutionary equation:

$$
\left\{\begin{array}{l}
\Phi^{\prime}(t)+(\mathcal{A}+\mathcal{B}) \Phi(t)=0, \quad t>0, \\
\Phi(0)=\Phi_{0}=\left(\varphi_{0}, \varphi_{1}, \psi_{0}, \psi_{1}, \omega_{0}, \omega_{1}, \theta_{0}, q_{0}, z_{0}\right)^{T}
\end{array}\right.
$$

where $\mathcal{A}$ is a linear operator defined by

$$
\mathcal{A} \Phi=\left(\begin{array}{c}
-u \\
-\frac{k}{\rho_{1}}\left(\varphi_{x}+\psi+l \omega\right)_{x}-\frac{k_{0} l}{\rho_{1}}\left(\omega_{x}-l \varphi\right)+\frac{|\mu|}{\rho_{1}} u+\frac{\mu}{\rho_{1}} z(\cdot, 1) \\
-v \\
-\frac{b}{\rho_{2}} \psi_{x x}+\frac{b}{\rho_{2}}\left(\varphi_{x}+\psi+l \omega\right)+\frac{\gamma}{\rho_{2}} \theta_{x} \\
-w \\
\frac{k_{0}}{\rho_{1}}\left(\omega_{x}-l \varphi\right)_{x}+\frac{k l}{\rho_{1}}\left(\varphi_{x}+\psi+l \omega\right) \\
\frac{1}{\rho_{3}} q_{x}+\frac{\gamma}{\rho_{3}} v_{x} \\
\frac{\beta}{\tau} q+\frac{1}{\tau} \theta_{x} \\
\frac{1}{\tau_{0}} z_{\rho}
\end{array}\right)
$$


and the operator $\mathcal{B}: D(\mathcal{B})=\mathcal{H} \rightarrow \mathcal{H}$ is defined by

$$
\mathcal{B} \Phi=\frac{|\mu|}{\rho_{1}}\left(\begin{array}{c}
0 \\
-u \\
0 \\
0 \\
0 \\
0 \\
0
\end{array}\right) .
$$

We give the following spaces:

$$
\begin{aligned}
& H_{*}^{1}(0,1)=\left\{f \in H^{1}(0,1): f(0)=0\right\}, \\
& \tilde{H}_{*}^{1}(0,1)=\left\{f \in H^{1}(0,1): f(1)=0\right\}, \\
& H_{*}^{2}(0,1)=H^{2}(0,1) \cap H_{*}^{1}(0,1), \\
& \tilde{H}_{*}^{2}(0,1)=H^{2}(0,1) \cap \tilde{H}_{*}^{1}(0,1),
\end{aligned}
$$

and the energy space:

$$
\begin{aligned}
\mathcal{H}= & H_{*}^{1}(0,1) \times L^{2}(0,1) \times \tilde{H}_{*}^{1}(0,1) \times L^{2}(0,1) \times \tilde{H}_{*}^{1}(0,1) \\
& \times L^{2}(0,1) \times L^{2}(0,1) \times L^{2}(0,1) \times L^{2}((0,1) \times(0,1)),
\end{aligned}
$$

equipped with the inner product

$$
\begin{aligned}
(\Phi, \tilde{\Phi})_{\mathcal{H}}= & k \int_{0}^{1}\left(\varphi_{x}+\psi+l \omega\right)\left(\tilde{\varphi}_{x}+\tilde{\psi}+l \tilde{\omega}\right) d x+k_{0} \int_{0}^{1}\left(\omega_{x}-l \varphi\right)\left(\tilde{\omega}_{x}-l \tilde{\varphi}\right) d x \\
& +\rho_{1} \int_{0}^{1} u \tilde{u} d x+b \int_{0}^{1} \psi_{x} \tilde{\psi}_{x} d x+\rho_{2} \int_{0}^{1} v \tilde{v} d x \\
& +\rho_{1} \int_{0}^{1} \omega \tilde{\omega} d x+\rho_{3} \int_{0}^{1} \theta \tilde{\theta} d x+\tau \int_{0}^{1} q \tilde{q} d x+\tau_{0}|\mu| \int_{0}^{1} \int_{0}^{1} z \tilde{z} d \rho d x .
\end{aligned}
$$

$\mathcal{H}$ is a Hilbert space for $l$ small enough. In this case, the above inner product is equivalent to the natural inner product defined on $\mathcal{H}$. To this end, the operator $\mathcal{A}$ with its domain is

$$
D(\mathcal{A})=\left\{\begin{array}{c}
\Psi \in \mathcal{H} \mid \varphi \in H_{*}^{2}(0,1) ; \psi, \omega \in \tilde{H}_{*}^{2}(0,1) ; u, \theta \in H_{*}^{1}(0,1), \\
v, w, q \in \tilde{H}_{*}^{1}(0,1) ; \varphi_{x}(1)=0, \psi_{x}(0)=\omega_{x}(0)=0, \\
z, z_{\rho} \in L^{2}\left((0,1), L^{2}(0,1)\right), z(x, 0)=\varphi(x)
\end{array}\right\} .
$$

In what follows, we have the well-posedness result of problem (2.2).

Theorem 2.1. Assume that $\Phi_{0} \in \mathcal{H}$, then problem (2.2) exists a unique solution $U \in$ $C\left(\mathbb{R}^{+}, \mathcal{H}\right)$. Moreover, if $\Phi_{0} \in D(\mathcal{A})$ then $\Phi \in C\left(\mathbb{R}^{+}, D(\mathcal{A})\right) \cap C^{1}\left(\mathbb{R}^{+}, \mathcal{H}\right)$.

Proof. It is easy to see that $D(\mathcal{A})$ is dense in $\mathcal{H}$. For $\Phi=(\varphi, u, \psi, v, \omega, w, \theta, q, z)^{T} \in D(\mathcal{A})$, a direct computation gives that

$$
(\mathcal{A} \Phi, \Phi)_{\mathcal{H}}=|\mu| \int_{0}^{1} u^{2} d x+\beta \int_{0}^{1} q^{2} d x+\mu \int_{0}^{1} u z(\cdot, 1) d x+|\mu| \int_{0}^{1} \int_{0}^{1} z z_{\rho} d \rho d x .
$$

By using Young's inequality, the third term in the right hand side of (2.3) gives

$$
-\mu \int u z(\cdot, 1) d x \leq \frac{|\mu|}{2} \int_{0}^{l} z^{2}(\cdot, 1) d x+\frac{|\mu|}{2} \int_{0}^{1} u^{2} d x,
$$

which implies that

$$
\mu \int_{0}^{1} u z(0,1) d x \geq-\frac{|\mu|}{2} \int_{0}^{l} z^{2}(\cdot, 1) d x-\frac{|\mu|}{2} \int_{0}^{1} u^{2} d x .
$$


Also, using integration by parts and the fact that $z(x, 0)=u(x)$, the last term in the right-hand side of (2.3) gives

$$
\int_{0}^{1} \int_{0}^{1} z z_{\rho} d \rho d x=\frac{1}{2} \int_{0}^{1} z^{2}(\cdot, 1) d x-\frac{1}{2} \int_{0}^{1} u^{2} d x .
$$

Consequently, (2.3) yields

$$
(\mathcal{A} \Phi, \Phi)_{\mathcal{H}} \geq \beta \int_{0}^{1} q^{2} d x .
$$

Hence $\mathcal{A}$ is monotone. Next, we will prove that the operator $\mathcal{J}+\mathcal{A}$ is surjective.

For all $G=\left(g_{1}, g_{2}, g_{3}, g_{4}, g_{5}, g_{6}, g_{7}, g_{8}, g_{9}\right)^{T} \in \mathcal{H}$, we solve the equation

$$
(\mathcal{J}+\mathcal{A}) \Phi=G .
$$

That is

$$
\left\{\begin{array}{l}
-u+\varphi=g_{1} \in H_{*}^{1}(0,1) \\
-k\left(\varphi_{x}+\psi+l \omega\right)_{x}-k_{0} l\left(\omega_{x}-l \varphi\right)+\left(|\mu|+\rho_{1}\right) u+\mu z(\cdot, 1)=\rho_{1} g_{2} \in L^{2}(0,1), \\
-v+\psi=g_{3} \in \tilde{H}_{*}^{1}(0,1) \\
-b \psi_{x x}+k\left(\varphi_{x}+\psi+l \omega\right)+\gamma \theta_{x}+\rho_{2} v=\rho_{2} g_{4} \in L^{2}(0,1), \\
-w+\omega=g_{5} \in \tilde{H}_{*}^{1}(0,1) \\
-k_{0}\left(\omega_{x}-l \varphi\right)_{x}+k l\left(\varphi_{x}+\psi+l \omega\right)+\rho_{1} w=\rho_{1} g_{6} \in L^{2}(0,1), \\
q_{x}+\gamma v_{x}+\rho_{3} \theta=\rho_{3} g_{7} \in L^{2}(0,1) \\
(\beta+\tau) q+\theta_{x}=\tau g_{8} \in L^{2}(0,1) \\
z_{\rho}+\tau_{0} z=\tau_{0} g_{9} \in L^{2}((0,1) \times(0,1)) .
\end{array}\right.
$$

From $(2.5)_{8}$, we know that

$$
\theta=\tau \int_{0}^{x} g_{8}(f) d y-(\beta+\tau) \int_{0}^{x} q(y) d y,
$$

which conclude $\theta(0, t)=0$. Inserting $u=\varphi-g_{1}, v=\psi-g_{3}, w=\omega-g_{5}$, the last equation in (2.5) together with the fact that $z(x, 0)=u(x)$, one has

$$
z(x, \rho)=\varphi(x) e^{-\tau_{0} \rho}-e^{-\tau_{0} \rho} g_{1}(x)+\tau_{0} e^{-\tau_{0} \rho} \int_{0}^{\rho} s^{\tau_{0} s} g_{9}(x, s) d s .
$$

It can be easily shown that $\varphi, \psi, \omega$ and $q$ satisfy

$$
\left\{\begin{array}{l}
-k\left(\varphi_{x}+\psi+l \omega\right)_{x}-k_{0} l\left(\omega_{x}-l \varphi\right)+\left(|\mu|+\rho_{1}+\mu e^{-\tau_{0}}\right) \varphi=h_{1} \in L^{2}(0,1), \\
-b \psi_{x x}+k\left(\varphi_{x}+\psi+l \omega\right)+\rho_{2} \psi-\gamma(\beta+\tau) q=h_{2} \in L^{2}(0,1), \\
-k_{0}\left(\omega_{x}-l \varphi\right)_{x}+k l\left(\varphi_{x}+\psi+l \omega\right)+\rho_{1} w=h_{3} \in L^{2}(0,1) \\
-q_{x}+\rho_{3}(\beta+\tau) \int_{0}^{s} q(y) d y-\gamma \psi_{x}=h_{4} \in L^{2}(0,1)
\end{array}\right.
$$

where

$$
\begin{aligned}
& h_{1}=\left(\rho_{1}+|\mu|+\mu\right) g_{1}+\rho_{1} g_{2}-\mu \tau_{0} e^{-\tau_{0}} \int_{0}^{1} e^{\tau_{0} s} g_{9}(x, s) d s, \\
& h_{2}=\rho_{2}\left(g_{3}+g_{4}\right)-\tau \gamma g_{8}, \\
& h_{3}=\rho_{1}\left(g_{5}+g_{6}\right) \\
& h_{4}=-\gamma g_{3 x}-\rho_{3}\left(g_{7}-\tau \int_{0}^{x} g_{8}(y) d y\right) .
\end{aligned}
$$

The variational formulation corresponding to (2.7) takes the form

$$
B((\varphi, \psi, \omega, q),(\tilde{\varphi}, \tilde{\psi}, \tilde{\omega}, \tilde{q}))=F(\tilde{\varphi}, \tilde{\psi}, \tilde{\omega}, \tilde{q}),
$$


where $B:\left[H_{*}^{1}(0,1) \times \tilde{H}_{*}^{1}(0,1) \times \tilde{H}_{*}^{1}(0,1) \times L^{2}(0,1)\right]^{2} \rightarrow \mathbb{R}$ is the bilinear form defined by

$$
\begin{aligned}
& B((\varphi, \psi, \omega, q),(\tilde{\varphi}, \tilde{\psi}, \tilde{\omega}, \tilde{q})) \\
= & k \int_{0}^{1}\left(\varphi_{x}+\psi+l \omega\right)(\tilde{\varphi}+\tilde{\psi}+l \tilde{\omega}) d x+(\beta+\tau) \int_{0}^{1} q \tilde{q} d x+b \int_{0}^{1} \psi_{x} \tilde{\psi}_{x} d x \\
& +\rho_{2} \int_{0}^{1} \psi \tilde{\psi} d x-\gamma(\beta+\tau) \int_{0}^{1} q \tilde{\psi} d x+\gamma(\beta+\tau) \int_{0}^{1} \psi \tilde{q} d x+\rho_{1} \int_{0}^{1} \varphi \tilde{\varphi} d x \\
& +\rho_{1} \int_{0}^{1} \omega \tilde{\omega} d x+k_{0} \int_{0}^{1}\left(\omega_{x}-l \varphi\right)\left(\tilde{\omega}_{x}-l \tilde{\varphi}\right) d x \\
& +\rho_{3}(\beta+\tau)^{2} \int_{0}^{1}\left(\int_{0}^{x} q(y) d y \int_{0}^{x} \tilde{q}(y) d y\right) d x,
\end{aligned}
$$

and $F:\left[H_{*}^{1}(0,1) \times \tilde{H}_{*}^{1}(0,1) \times \tilde{H}_{*}^{1}(0,1) \times L^{2}(0,1)\right] \rightarrow R$ is the linear functional given by

$$
F(\tilde{\varphi}, \tilde{\psi}, \tilde{\omega}, \tilde{q})=\int_{0}^{1} h_{1} \tilde{\varphi} d x+\int_{0}^{1} h_{2} \tilde{\psi} d x+\int_{0}^{1} h_{3} \tilde{\omega} d x+(\beta+\tau) \int_{0}^{1} h_{4} \int_{0}^{x} \tilde{q}(y) d y d x .
$$

Now, for $V=H_{*}^{1}(0,1) \times \tilde{H}_{*}^{1}(0,1) \times \tilde{H}_{*}^{1}(0,1) \times L^{2}(0,1)$ equipped with the norm

$$
\|(\varphi, \psi, \omega, q)\|_{V}=\left\|\left(\varphi_{x}+\psi+l \omega\right)\right\|_{2}^{2}+\left\|\omega_{x}-l \varphi\right\|_{2}^{2}+\left\|\psi_{x}\right\|_{2}^{2}+\|q\|_{2}^{2},
$$

and combining with

$$
\int_{0}^{1}\left(\varphi_{x}^{2}+\psi_{x}^{2}+\omega_{x}^{2}\right) d x \leq c \int_{0}^{1}\left(\left(\varphi_{x}+\psi+l \omega\right)^{2}+\left(\omega_{x}-l \varphi\right)^{2}+\psi_{x}^{2}\right) d x
$$

for $l$ small enough, it follows that $B$ and $F$ are bounded. Furthermore, using the definition of $B$, we get

$$
\begin{aligned}
B((\varphi, \psi, \omega, q),(\varphi, \psi, \omega, q))= & k \int_{0}^{1}\left(\varphi_{x}+\psi+l \omega\right)^{2} d x+(\beta+\tau) \int_{0}^{1} q^{2} d x+b \int_{0}^{1} \psi_{x}^{2} d x \\
& +\rho_{2} \int_{0}^{1} \psi^{2} d x+\rho_{1} \int_{0}^{1} \varphi^{2} d x+\rho_{1} \int_{0}^{1} \omega^{2} d x \\
& +k_{0} \int_{0}^{1}\left(\omega_{x}-l \varphi\right)^{2} d x+\rho_{3}(\beta+\tau)^{2} \int_{0}^{1}\left(\int_{0}^{x} q(y) d y\right)^{2} d x \\
\geq & c\|(\varphi, \psi, \omega, q)\|_{V}^{2} .
\end{aligned}
$$

Thus, $B$ is coercive. Consequently, Lax-Milgram Lemma provides that system (2.7) has a unique solution $\varphi \in H_{*}^{1}(0,1), \psi \in \tilde{H}_{*}^{1}(0,1), \omega \in \tilde{H}_{*}^{1}(0,1), q \in L^{2}(0,1)$. Substituting $\varphi, \psi$, $\omega, q$ into $(2.5)_{1},(2.5)_{3},(2.5)_{5}$ and $(2.5)_{8}$ respectively, we get $u \in H_{*}^{1}(0,1), v \in \tilde{H}_{*}^{1}(0,1)$, $w \in \tilde{H}_{*}^{1}(0,1), \theta \in H_{*}^{1}(0,1)$.

If $(\tilde{\psi}, \tilde{\omega}, \tilde{q}) \equiv(0,0,0) \in \tilde{H}_{*}^{1}(0,1) \times \tilde{H}_{*}^{1}(0,1) \times L^{2}(0,1)$, then $(2.8)$ reduces to

$$
k \int_{0}^{1}\left(\varphi_{x}+\psi+l \omega\right) \tilde{\varphi}_{x} d x-k_{0} l \int_{0}^{1}\left(\omega_{x}-l \varphi\right) \tilde{\varphi} d x+\rho_{1} \int_{0}^{1} \varphi \tilde{\varphi} d x=\int_{0}^{1} h_{1} \tilde{\varphi} d x
$$

for all $\tilde{\varphi}$ in $H_{*}^{1}(0,1)$, which implies

$$
-k \varphi_{x x}=k \psi_{x}+l\left(k+k_{0}\right) \omega_{x}-\left(k_{0} l^{2}+\rho_{1}\right) \varphi+h_{1} \in L^{2}(0,1) .
$$

Consequently, by the regularity theory for the linear elliptic equations, we obtain

$$
\varphi \in H_{*}^{2}(0,1) .
$$

Moreover, (2.9) is also true for any $\phi \in C^{1}([0,1]), \phi(0)=0$ which is in $H_{*}^{1}(0,1)$. Hence, taking any $\phi \in C^{1}([0,1]), \phi(0)=0$, one has

$$
k \int_{0}^{1} \varphi_{x} \phi_{x} d x-\int_{0}^{1}\left(k \psi_{x}+l\left(k+k_{0}\right) \omega_{x}-\left(k_{0} l^{2}+\rho_{1}\right) \varphi+h_{1}\right) \phi d x=0
$$


Thus, using integration by parts and taking into account (2.10), we get

$$
\varphi_{x}(1) \phi(1)=0, \forall \phi \in C^{1}([0,1]), \phi(0)=0 .
$$

Therefore,

Similarly, we get

$$
\varphi_{x}(1)=0
$$

$$
\left\{\begin{array}{l}
-b \psi_{x x}=-k \varphi_{x}-(k+\rho-2) \psi-l k \omega-\gamma(\beta+\tau) q+h_{2} \in L^{2}(0,1), \\
-k \omega_{x x}=-l\left(k+k_{0}\right) \varphi_{x}-l k \psi+\left(\rho_{1}+l^{2} k_{0}\right) \omega+h_{3} \in L^{2}(0,1), \\
-q_{x}=\gamma \psi_{x}-(\beta+\tau) \rho_{3} \int_{0}^{x} q(y) d y+h_{4} \in L^{2}(0,1) .
\end{array}\right.
$$

Thus, we have

$$
\psi, \omega \in \tilde{H}_{*}^{2}(0,1), q \in \tilde{H}_{*}^{1}(0,1), \omega_{x}(0)=\psi_{x}(0)=0 .
$$

Hence, there exists a unique $\Phi \in D(\mathcal{A})$ such that (2.4) is satisfied, which conclude that the operator $\mathcal{A}$ is maximal. With this, it is easy to obtain that $\mathcal{A}$ is a maximal monotone operator. On the other hand, it is obvious that operator $\mathcal{B}$ is Lipschitz continuous. Consequently, $\mathcal{A}+\mathcal{B}$ is the infinitesimal generator of a linear contraction $C_{0}$-semigroup on $\mathcal{H}$. This completes the proof (see [26] and [11]).

\section{Exponential stability}

In this section, we state and prove our stability result for the solution of system (2.1) by using the multiplier technique. We first introduce the following energy functional:

$$
\begin{aligned}
E(t)= & \frac{1}{2} \int_{0}^{1}\left[\rho_{1} \varphi_{t}^{2}+\rho_{2} \psi_{t}^{2}+\rho_{1} \omega_{t}^{2}+b \psi_{x}^{2}+\rho_{3} \theta^{2}+\tau q^{2}+k\left(\varphi_{x}+\psi+l \omega\right)^{2}\right] d x \\
& +\frac{1}{2} \int_{0}^{1}\left[k_{0}\left(\omega_{x}-l \varphi\right)^{2}+|\mu| \tau_{0} \int_{0}^{1} z^{2}(x, \rho, t) d \rho\right] d x
\end{aligned}
$$

Our main result of this section reads as follows.

Theorem 3.1. Let $(\varphi, \psi, \omega, \theta, q, z)$ be the solution of (2.1), assume that $k=k_{0}$ and

$$
\xi:=\left(\tau-\frac{\rho_{1}}{k \rho_{3}}\right)\left(\frac{\rho_{2}}{b}-\frac{\rho_{1}}{k}\right)-\frac{\tau \rho_{1} \gamma^{2}}{b k \rho_{3}}=0 .
$$

Then for $|\mu|$ small enough, the energy functional (3.1) satisfies

$$
E(t) \leq k_{1} e^{-k_{2} t}, \forall t \geq 0
$$

where $k_{1}, k_{2}$ are two positive constants.

We need the following lemmas to show that the associated energy non-increase in time.

Lemma 3.2. Let $(\varphi, \psi, \omega, \theta, q, z)$ be the solution of (2.1), the energy functional defined by (3.1) satisfies

$$
E^{\prime}(t)=-\beta \int_{0}^{1} q^{2} d x+|\mu| \int_{0}^{1} \varphi_{t}^{2} d x
$$

Proof. $(2.1)_{1},(2.1)_{2},(2.1)_{3},(2.1)_{4}$ and $(2.1)_{5}$, by multiplying $\varphi_{t}, \psi_{t}, \omega_{t}, \theta$ and $q$ respectively, then integrating over $(0,1)$ and summing up, using the boundary conditions, we get

$$
\begin{aligned}
& \frac{1}{2} \frac{d}{d t}\left\{\rho_{1} \int_{0}^{1} \varphi_{t}^{2} d x+\rho_{2} \int_{0}^{1} \psi_{t}^{2} d x+\rho_{1} \int_{0}^{1} \omega_{t}^{2} d x+b \int_{0}^{1} \psi_{x}^{2} d x+\rho_{3} \int_{0}^{1} \theta^{2} d x+\tau \int_{0}^{1} q^{2} d x\right\} \\
& +\frac{1}{2} \frac{d}{d t}\left\{\int_{0}^{1} k_{0}\left(\omega_{x}-l \varphi\right)^{2} d x+k \int_{0}^{1}\left(\varphi_{x}+\psi+l \omega\right)^{2} d x+|\mu| \tau_{0} \int_{0}^{1} \int_{0}^{1} z^{2}(x, \rho, t) d \rho d x\right\}
\end{aligned}
$$




$$
=-\beta \int_{0}^{1} q^{2} d x+|\mu| \int_{0}^{1} \varphi_{t}^{2} d x .
$$

Now, multiplying $(2.1)_{6}$ by $|\mu| z$ and integrating over $(0,1) \times(0,1)$, bearing in mind $z(x, 0, t)=\varphi_{t}(x, t)$, we obtain

$$
\frac{|\mu| \tau_{0}}{2} \frac{d}{d t} \int_{0}^{1} \int_{0}^{1} z^{2}(x, \rho, t) d \rho d x=\frac{|\mu|}{2} \int_{0}^{1} \varphi_{t}^{2} d x-\frac{|\mu|}{2} \int_{0}^{1} z^{2}(x, 1, t) d x .
$$

The result follows by the combination of (3.4)-(3.5) and Young's inequality.

Lemma 3.3. Let $(\varphi, \psi, \omega, \theta, q, z)$ be the solution of (2.1). The functional

$$
F_{1}(t)=-\rho_{1} \int_{0}^{1}\left(\varphi \varphi_{t}+\omega \omega_{t}\right) d x
$$

satisfies

$$
\begin{aligned}
F_{1}^{\prime}(t) \leq & -\rho_{1} \int_{0}^{1} \varphi_{t}^{2} d x-\rho_{1} \int_{0}^{1} \omega_{t}^{2} d x+C \int_{0}^{1} \psi_{x}^{2} d x+k_{0} \int_{0}^{1}\left(\omega_{x}-l \varphi\right)^{2} d x \\
& +C\left(\varepsilon_{1}\right) \int_{0}^{1}\left(\varphi_{x}+\psi+l \omega\right)^{2} d x+\varepsilon_{1} \int_{0}^{1} z^{2}(x, 1, t) d x
\end{aligned}
$$

for all $\varepsilon_{1}>0$.

Proof. By differentiating $F_{1}$ and using $(2.1)_{1}$ and $(2.1)_{3}$, we conclude that

$$
\begin{aligned}
& F_{1}^{\prime}(t) \\
= & -\rho_{1} \int_{0}^{1} \varphi_{t}^{2} d x-\rho_{1} \int_{0}^{1} \omega_{t}^{2} d x-\int_{0}^{1} \varphi\left(k\left(\varphi_{x}+\psi+l \omega\right)_{x}+l k_{0}\left(\omega_{x}-l \varphi\right)-\mu z(x, 1, t)\right) d x \\
& -\rho \int_{0}^{1} \omega\left(k_{0}\left(\omega_{x}-l \varphi\right)_{x}-l k\left(\varphi_{x}+\psi+l \omega\right)\right) d x \\
= & -\rho_{1} \int_{0}^{1} \varphi_{t}^{2} d x-\rho_{1} \int_{0}^{1} \omega_{t}^{2} d x+k \int_{0}^{1}\left(\varphi_{x}+\psi+l \omega\right)^{2} d x-k \int_{0}^{1} \psi\left(\varphi_{x}+\psi+l \omega\right) d x \\
& +k_{0} \int_{0}^{1}\left(\omega_{x}-l \varphi\right)^{2} d x-\mu \int_{0}^{1} \varphi z(x, 1, t) d x .
\end{aligned}
$$

Using Young's and Poincaré inequalities, (3.6) is established.

Lemma 3.4. Let $(\varphi, \psi, \omega, \theta, q, z)$ be the solution of (2.1). The functional

$$
F_{2}(t)=\rho_{2} \int_{0}^{1} \psi \psi_{t} d x
$$

satisfies the estimate

$$
F_{2}^{\prime}(t) \leq-\frac{b}{2} \int_{0}^{1} \psi_{x}^{2} d x+\rho_{2} \int_{0}^{1} \psi_{t}^{2} d x+\frac{k^{2}}{b} \int_{0}^{1}\left(\varphi_{x}+\psi-l \omega\right)^{2} d x+C \int_{0}^{1} \theta^{2} d x
$$

Proof. Taking the derivative of $F_{2}$ with respect to $t$ and using $(2.1)_{2}$, it follows that

$$
F_{2}^{\prime}(t)=\rho_{2} \int_{0}^{1} \psi_{x}^{2} d x-\int_{0}^{1} \psi\left(b \psi_{x x}-k\left(\varphi_{x}-\psi+l \omega\right)-\gamma \theta_{x}\right) d x .
$$

Using Young's and Poincaré inequalities, we obtain (3.7).

Lemma 3.5. Let $(\varphi, \psi, \omega, \theta, q, z)$ be the solution of (2.1). The functional

$$
F_{3}(t)=-\frac{\rho_{2} \rho_{3}}{\gamma} \int_{0}^{1} \theta \int_{0}^{x} \psi_{t}(y) d y d x
$$

satisfies

$$
F_{3}^{\prime}(t) \leq-\frac{\rho_{2}}{2} \int_{0}^{1} \psi_{t}^{2} d x+\varepsilon_{3} \int_{0}^{1}\left(\varphi_{x}+\psi+l \omega\right)^{2} d x+\varepsilon_{3} \int_{0}^{1} \psi_{x}^{2} d x+C\left(\varepsilon_{3}\right) \int_{0}^{1} \theta^{2} d x
$$




$$
+C \int_{0}^{1} q^{2} d x
$$

for all $\varepsilon_{3}>0$.

Proof. By differentiating $F_{3}$ and using $(2.1)_{2}$ and $(2.1)_{4}$, we get

$$
\begin{aligned}
F_{3}^{\prime}(t)= & \frac{\rho_{2}}{\gamma} \int_{0}^{1}\left(q_{x}+\gamma \psi_{t x}\right) \int_{0}^{x} \psi_{t}(y) d y d x-\frac{\rho_{3}}{\gamma} \int_{0}^{1} \theta \int_{0}^{x}\left(b \psi_{x x}-k\left(\varphi_{x}+\psi+l \omega\right)\right) d y d x \\
= & -\rho_{2} \int_{0}^{1} \psi_{t}^{2} d x-\frac{\rho_{2}}{\gamma} \int_{0}^{1} q \psi_{t} d x+\rho_{3} \int_{0}^{1} \theta^{2} d x-\frac{b \rho_{3}}{\gamma} \int_{0}^{1} \theta \psi_{x} d x+\frac{b \rho_{3}}{\gamma} \int_{0}^{1} \theta \psi_{x} d x \\
& +\frac{k \rho_{3}}{\gamma} \int_{0}^{1}\left(\varphi_{x}+\psi+l \omega\right) \int_{0}^{x} \theta(y) d y d x
\end{aligned}
$$

The result thanks to Young's inequality.

Lemma 3.6. Let $(\varphi, \psi, \omega, \theta, q, z)$ be the solution of (2.1). The functional

satisfies

$$
F_{4}(t)=\tau \rho_{3} \int_{0}^{1} \theta \int_{0}^{x} q(y) d y d x,
$$

$$
F_{4}^{\prime}(t) \leq-\frac{\rho_{3}}{2} \int_{0}^{1} \theta^{2} d x+\varepsilon_{4} \int_{0}^{1} \psi_{t}^{2} d x+C\left(\varepsilon_{4}\right) \int_{0}^{1} q^{2} d x
$$

for all $\varepsilon_{4}>0$.

Proof. Differentiating $F_{4}$ with respect to $t$, using $(2.1)_{4}$ and $(2.1)_{5}$, one has

$$
\begin{aligned}
F_{4}^{\prime}(t) & =\tau \int_{0}^{1}\left(-q_{x}-\gamma \psi_{t x}\right) \int_{0}^{x} q(y) d y d x+\rho_{3} \int_{0}^{1} \theta \int_{0}^{x}\left(-\beta q-\theta_{x}\right) d y d x \\
& =-\rho_{3} \int_{0}^{1} \theta^{2} d x+\tau \int_{0}^{1} q^{2} d x+\tau \gamma \int_{0}^{1} q \psi_{t} d x-\beta \rho_{3} \int_{0}^{1} \theta \int_{0}^{x} q y d y d x .
\end{aligned}
$$

Then, we use Cauchy-Schwarz and Young's inequalities with $\varepsilon_{4}>0$ to obtain (3.9).

Lemma 3.7. Let $(\varphi, \psi, \omega, \theta, q, z)$ be the solution of (2.1). The functional

satisfies

$$
F_{5}(t)=-\rho_{1} \int_{0}^{1} \varphi_{t}\left(\omega_{x}-l \varphi\right) d x-\rho_{1} \int_{0}^{1} \omega_{t}\left(\varphi_{x}+\psi+l \omega\right) d x
$$

$$
\begin{aligned}
F_{5}^{\prime}(t) \leq & -\frac{l k_{0}}{2} \int_{0}^{1}\left(\omega_{x}-l \varphi\right)^{2} d x-\frac{l \rho_{1}}{2} \int_{0}^{1} \omega_{t}^{2} d x+l \rho_{1} \int_{0}^{1} \varphi_{t}^{2} d x+l k \int_{0}^{1}\left(\varphi_{x}+\psi+l \omega\right)^{2} d x \\
& +\frac{\rho_{1}}{2 l} \int_{0}^{1} \psi_{t}^{2} d x+2 l k_{0} \int_{0}^{1} \mu^{2} z^{2}(x, 1, t) d x
\end{aligned}
$$

for all $\varepsilon_{5}>0$.

Proof. Differentiating $F_{5}$ with respect to $t$, using $(2.1)_{1}$ and $(2.1)_{3}$, it follows that

$$
\begin{aligned}
F_{5}^{\prime}(t)= & -\int_{0}^{1}\left(k\left(\varphi_{x}+\psi+l \omega\right)_{x}+l k_{0}(\omega-l \varphi)-\mu z(x, 1, t)\right)\left(\omega_{x}-l \varphi\right) d x \\
& -\int_{0}^{1}\left(k_{0}\left(\omega_{x}-l \varphi\right)_{x}-l k\left(\varphi_{x}+\psi+l \omega\right)\right)\left(\varphi_{x}+\psi+l \omega\right) d x \\
& -\rho_{1} \int_{0}^{1} \varphi_{t}\left(\omega_{x}-l \varphi\right)_{t} d x-\rho_{1} \int_{0}^{1} \omega_{t}\left(\varphi_{x}+\psi+l \omega\right)_{t} d x \\
= & -l k_{0} \int_{0}^{1}\left(\omega_{x}-l \varphi\right)^{2} d x+l k \int_{0}^{1}\left(\varphi_{x}+\psi+l \omega\right)^{2} d x-\rho_{1} \int_{0}^{1} \omega_{t}^{2} d x+l \rho_{1} \int_{0}^{1} \varphi_{t}^{2} d x \\
& -\rho_{1} \int_{0}^{1} \psi_{t} \omega_{t} d x+\int_{0}^{1} \mu z(x, 1, t)\left(\omega_{x}-l \varphi\right) d x .
\end{aligned}
$$


(3.10) follows Young's inequality with the fact that $k=k_{0}$.

Lemma 3.8. Let $(\varphi, \psi, \omega, \theta, q, z)$ be the solution of (2.1). The functional

$$
F_{6}(t)=\tau_{0} \int_{0}^{1} \int_{0}^{1} e^{-\tau_{0} \rho} z(x, \rho, t) d \rho d x
$$

satisfies

$$
F_{6}^{\prime}(t) \leq-m\left(\int_{0}^{1} z^{2}(x, 1, t) d x+\tau_{0} \int_{0}^{1} \int_{0}^{1} z^{2}(x, \rho, t) d \rho d x\right)+\int_{0}^{1} \varphi_{t}^{2} d x,
$$

where $m=\min \left\{e^{-\tau_{0}}, e^{-\tau_{0} \rho}\right\}$.

Proof. Similarly computation, using $(2.1)_{6}$, we have

$$
\begin{aligned}
F_{6}^{\prime}(t)= & \frac{d}{d \rho} \int_{0}^{1} \int_{0}^{1} e^{-\tau_{0} \rho} z^{2}(x, \rho, t) d \rho d x-\tau_{0} \int_{0}^{1} \int_{0}^{1} e^{-\tau_{0} \rho} z^{2}(x, \rho, t) d \rho d x \\
& -\int_{0}^{1}\left[e^{-\tau_{0}} z^{2}(x, 1, t)-z^{2}(x, 0, t)\right] d x-\tau_{0} \int_{0}^{1} \int_{0}^{1} e^{-\tau_{0} \rho} z^{2}(x, \rho, t) d \rho d x .
\end{aligned}
$$

It is obvious that result (3.11).

Lemma 3.9. Let $(\varphi, \psi, \omega, \theta, q, z)$ be the solution of (2.1). The functional

$$
F_{7}(t)=-\rho_{1} \int_{0}^{1}\left(\omega_{x}-l \varphi\right) \int_{0}^{x} \omega_{t}(y) d y d x-\rho_{1} \int_{0}^{1} \varphi_{t} \int_{0}^{x}\left(\varphi_{x}+\psi+l \omega\right)(y) d y d x
$$

satisfies

$$
\begin{aligned}
F_{7}^{\prime}(t) \leq & -\frac{\rho_{1}}{2} \int_{0}^{1} \varphi_{t}^{2} d x-k_{0} \int_{0}^{1}\left(\omega_{x}-l \varphi\right)^{2} d x+\left(k+\frac{1}{2}\right) \int_{0}^{1}\left(\varphi_{x}+\psi+l \omega\right)^{2} d x \\
& +\rho_{1} \int_{0}^{1} \omega_{t}^{2} d x+\frac{\rho_{1}}{2} \int_{0}^{1} \psi_{t}^{2} d x+\frac{1}{2} \mu^{2} \int_{0}^{1} z^{2}(x, 1, t) d x
\end{aligned}
$$

Proof. Differentiating $F_{7}$ with respect to $t$, using $(2.1)_{1}$ and $(2.1)_{3}$, we get

$$
\begin{aligned}
F_{7}^{\prime}(t)= & -\rho_{1} \int_{0}^{1}\left(\omega_{x}-l \varphi\right)_{t} \int_{0}^{x} \omega_{t}(y) d y d x-\rho_{1} \int_{0}^{1} \varphi_{t} \int_{0}^{1}\left(\varphi_{x}+\psi+l \omega\right)_{t}(y) d y d x \\
& -\int_{0}^{1}\left(\omega_{x}-l \varphi\right) \int_{0}^{x}\left(k_{0}\left(\omega_{x}-l \varphi\right)_{x}-l k\left(\varphi_{x}+\psi+l \omega\right)\right) d y d x \\
& -\int_{0}^{1}\left(k\left(\varphi_{x}+\psi+l \omega\right)_{x}+l k_{0}\left(\omega_{x}-l \varphi\right)-\mu z(x, 1, t)\right) \int_{0}^{x}\left(\varphi_{x}+\psi+l \omega\right)(y) d y d x \\
= & \rho_{1} \int_{0}^{1} \omega_{t}^{2} d x+\int_{0}^{1} \mu z(x, 1, t) \int_{0}^{x}\left(\varphi_{x}+\psi+l \omega\right)(y) d y d x \\
& -k_{0} \int_{0}^{1}\left(\omega_{x}-l \varphi\right)^{2} d x+l\left(k-k_{0}\right) \int_{0}^{1}\left(\omega_{x}-l \varphi\right) \int_{0}^{x}\left(\varphi_{x}+\psi+l \omega\right)(y) d y d x \\
& +k \int_{0}^{1}\left(\varphi_{x}+\psi+l \omega\right)^{2} d x-\rho_{1} \int_{0}^{1} \varphi_{t}^{2} d x-\rho_{1} \int_{0}^{1} \varphi_{t} \int_{0}^{x} \psi_{t}(y) d y d x .
\end{aligned}
$$

The result follows Young's and Cauchy-Schwarz inequalities with the fact that $k=k_{0}$.

Lemma 3.10. Let $(\varphi, \psi, \omega, \theta, q, z)$ be the solution of (2.1). The functional

$$
\begin{aligned}
F_{8}(t)= & \rho_{2} \int_{0}^{1} \psi_{t}\left(\varphi_{x}+\psi+l \omega\right) d x+\frac{b \rho_{1}}{k} \int_{0}^{1} \varphi_{t} \psi_{x} d x+\frac{b \rho_{3}}{\gamma}\left(\frac{\rho_{1}}{k}-\frac{\rho_{2}}{b}\right) \int_{0}^{1} \theta \varphi_{t} d x \\
& -\frac{b}{\gamma}\left(\frac{\rho_{1}}{k}-\frac{\rho_{2}}{b}\right) \int_{0}^{1} q\left(\varphi_{x}+\psi+l \omega\right) d x-\frac{b l^{2} \rho_{2}}{k_{0}} \int_{0}^{1} \psi_{t} \psi d x+\frac{b l \rho_{1}}{k_{0}} \int_{0}^{1} \omega_{t} \psi d x
\end{aligned}
$$

satisfies

$$
F_{8}^{\prime}(t) \leq-\frac{k}{2} \int_{0}^{1}\left(\varphi_{x}+\psi+l \omega\right)^{2} d x+\frac{2 b^{2} l^{2}}{k} \int_{0}^{1} \psi_{x}^{2} d x+C(\varepsilon) \int_{0}^{1} \psi_{t}^{2} d x+\varepsilon_{8} \int_{0}^{1} \omega_{t}^{2} d x
$$




$$
+C\left(\varepsilon_{8}\right) \int_{0}^{1} q^{2} d x+C\left(\varepsilon_{8}\right) \int_{0}^{1} \theta^{2} d x+\varepsilon_{8} \int_{0}^{1}\left(\omega_{x}-l \varphi\right)^{2} d x+C \int_{0}^{1} \mu z^{2}(x, 1, t) d x,
$$

for all $\varepsilon_{8}>0$.

Proof. A differentiation of above functional gives

$$
\begin{aligned}
& F_{8}^{\prime}(t) \\
& =\int_{0}^{1}\left(b \psi_{x x}-k\left(\varphi_{x}+\psi+l \omega\right)-\gamma \theta_{x}\right)\left(\varphi_{x}+\psi+l \omega\right) d x \\
& +\rho_{2} \int_{0}^{1} \psi_{t}\left(\varphi_{x}+\psi+l \omega\right) d x+\frac{b}{k} \int_{0}^{1}\left(k\left(\varphi_{x}+\psi+l \omega\right)_{x}+l k_{0}\left(\omega_{x}-l \varphi\right)-\mu z(x, 1, t)\right) \psi_{x} d x \\
& +\frac{b \rho_{1}}{k \gamma} \int_{0}^{1} \varphi_{x}\left(-\rho_{3} \theta_{t}-q_{x}\right) d x+\frac{b}{\gamma}\left(\frac{\rho_{1}}{k}-\frac{\rho_{2}}{b}\right) \int_{0}^{1}\left(-q_{x}-\gamma \psi_{t x}\right) \varphi_{t} d x \\
& +\frac{b \rho_{3}}{\gamma \rho_{1}}\left(\frac{\rho_{1}}{k}-\frac{\rho_{2}}{b}\right) \int_{0}^{1} \theta\left(k\left(\varphi_{x}+\psi+l \omega\right)_{x}+l k_{0}\left(\omega_{x}-l \varphi\right)-\mu z(x, 1, t)\right) d x \\
& -\frac{b}{\gamma \tau}\left(\frac{\rho_{1}}{k}-\frac{\rho_{2}}{b}\right) \int_{0}^{1}\left(-\beta q-\theta_{x}\right)\left(\varphi_{x}+\psi+l \omega\right) d x-\frac{b}{\gamma}\left(\frac{\rho_{1}}{k}-\frac{\rho_{2}}{b}\right) \int_{0}^{1} q\left(\varphi_{x}+\psi+l \omega\right)_{t} d x \\
& -\frac{b l^{2}}{k_{0}} \int_{0}^{1}\left(b \psi_{x x}-k\left(\varphi_{x}+\psi+l \omega\right)-\gamma \theta_{x}\right) \psi d x-\frac{b l^{2} \rho_{2}}{k_{0}} \int_{0}^{1} \psi_{t}^{2} d x \\
& +\frac{b l}{k_{0}} \int_{0}^{1}\left(k_{0}\left(\omega_{x}-l \varphi\right)_{x}-l k\left(\varphi_{x}+\psi+l \omega\right)\right) \psi d x+\frac{b l \rho_{1}}{k_{0}} \int_{0}^{1} \omega_{t} \psi_{t} d x \\
& =-k \int_{0}^{1}\left(\varphi_{x}+\psi+l \omega\right)^{2} d x+\left(\rho_{2}-\frac{b l^{2} \rho_{2}}{k_{0}}\right) \int_{0}^{1} \psi_{t}^{2} d x+\left(l \rho_{2}+\frac{b l \rho_{1}}{k_{0}}\right) \int_{0}^{1} \psi_{t} \omega_{t} d x \\
& +\left(-\gamma-\frac{\rho_{3} k b}{\gamma \rho_{1}}\left(\frac{\rho_{1}}{k}-\frac{\rho_{2}}{b}\right)+\frac{b}{\gamma \tau}\left(\frac{\rho_{1}}{k}-\frac{\rho_{2}}{b}\right)\right) \int_{0}^{1} \theta_{x}\left(\varphi_{x}+\psi+l \omega\right) d x \\
& +\frac{b}{\gamma}\left(\frac{\rho_{1}}{k}-\frac{\rho_{2}}{b}\right) \int_{0}^{1} q \psi_{t} d x-\frac{b l}{\gamma}\left(\frac{\rho_{1}}{k}-\frac{\rho_{2}}{b}\right) \int_{0}^{1} q \omega_{t} d x+\frac{b l k_{0} \rho_{3}}{r \rho_{1}}\left(\frac{\rho_{1}}{k}-\frac{\rho_{2}}{b}\right) \int_{0}^{1} \theta\left(\omega_{x}-l \varphi\right) d x \\
& -\frac{\gamma b l^{2}}{k_{0}} \int_{0}^{1} \theta \psi_{x} d x+\frac{b \beta}{\gamma \tau}\left(\frac{\rho_{1}}{k}-\frac{\rho_{2}}{b}\right) \int_{0}^{1} q\left(\varphi_{x}+\psi+l \omega\right) d x+\frac{b^{2} l^{2}}{k_{0}} \int_{0}^{1} \psi_{x}^{2} d x \\
& +b l\left(\frac{k_{0}}{k}-1\right) \int_{0}^{1} \psi_{x}\left(\omega_{x}-l \varphi\right) d x-\frac{b}{k} \int_{0}^{1} \mu z(x, 1, t) \psi_{x} d x-\frac{b \rho_{3}}{\gamma \rho_{1}}\left(\frac{\rho_{1}}{k}-\frac{\rho_{2}}{b}\right) \int_{0}^{1} \theta \mu z(x, 1, t) d x .
\end{aligned}
$$

Noting that $k=k_{0}$ and $\xi=0$, the above equation turns into

$F_{8}^{\prime}(t)$

$$
\begin{aligned}
& =-k \int_{0}^{1}\left(\varphi_{x}+\psi+l \omega\right)^{2} d x+\left(\rho_{2}-\frac{b l \rho_{2}}{k_{0}}\right) \int_{0}^{1} \psi_{t}^{2} d x+\left(l \rho_{2}+\frac{b l \rho_{1}}{k_{0}}\right) \int_{0}^{1} \psi_{t} \omega_{t} d x \\
& +\frac{b}{\gamma}\left(\frac{\rho_{1}}{k}-\frac{\rho_{2}}{b}\right) \int_{0}^{1} q \psi_{t} d x-\frac{b l}{\gamma}\left(\frac{\rho_{1}}{k}-\frac{\rho_{2}}{b}\right) \int_{0}^{1} q \omega_{t} d x+\frac{b l k_{0} \rho_{3}}{\gamma \rho_{1}}\left(\frac{\rho_{1}}{k}-\frac{\rho_{2}}{b}\right) \int_{0}^{1} \theta\left(\omega_{x}-l \varphi\right) d x \\
& -\frac{\gamma b l^{2}}{k_{0}} \int_{0}^{1} \theta \psi_{x} d x+\frac{b \beta}{\gamma \tau}\left(\frac{\rho_{1}}{k}-\frac{\rho_{2}}{b}\right) \int_{0}^{1} q\left(\varphi_{x}+\psi+l \omega\right) d x+\frac{b^{2} l^{2}}{k_{0}} \int_{0}^{1} \psi_{x}^{2} d x \\
& -\frac{b}{k} \int_{0}^{1} \mu z(x, 1, t) \psi_{x} d x-\frac{b \rho_{3}}{\gamma \rho_{1}}\left(\frac{\rho_{1}}{k}-\frac{\rho_{2}}{b}\right) \int_{0}^{1} \theta \mu z(x, 1, t) d x .
\end{aligned}
$$

Using Young's inequality, we get (3.13).

Now, we are ready to prove an exponential decay result under a smallness condition on the delay. 


\section{Proof of Theorem 3.1}

We define a Lyapunov functional

$$
\mathcal{L}(t):=N E(t)+\sum_{i=1}^{8} N_{i} F_{i}(t),
$$

which it is equivalent to the energy functional $E$. Now, gathering the estimates in Lemmas 3.3-3.10, we obtain

$$
\begin{aligned}
\mathcal{L}^{\prime}(t) \leq & -\left(N_{1} \rho_{1}+\frac{\rho_{1}}{2} N_{7}-\rho_{1} l N_{5}-N_{6}-\mu N\right) \int \varphi_{t}^{2} d x \\
& -\left(N_{1} \rho_{1}+\frac{l \rho_{1}}{2} N_{5}-\rho_{1} N_{7}-\varepsilon_{8} N_{8}\right) \int_{0}^{1} \omega_{t}^{2} d x \\
& -\left(\frac{b}{2} N_{2}-C N_{1}-\varepsilon_{3} N_{3}-\frac{2 b^{2} l^{2}}{k} N_{7}\right) \int_{0}^{1} \psi_{x}^{2} d x \\
& -\left(\frac{\rho_{3}}{2} N_{4}-C N_{2}-C\left(\varepsilon_{3}\right) N_{3}-C\left(\varepsilon_{8}\right) N_{8}\right) \int_{0}^{1} \theta^{2} d x \\
& -\left(\frac{\rho_{2}}{2} N_{3}-\rho_{2} N_{2}-\varepsilon_{4} N_{4}-\frac{\rho_{1}}{2 l} N_{5}-\frac{\rho_{1}}{2} N_{7}-C\left(\varepsilon_{8}\right) N_{8}\right) \int_{0}^{1} \psi_{t}^{2} d x \\
& -\left(\frac{l k_{0}}{2} N_{5}-k_{0} N_{1}+k_{0} N_{7}-\varepsilon_{8} N_{8}\right) \int_{0}^{1}\left(\omega_{x}-l \varphi\right)^{2} d x \\
& -\left(N \beta-C N_{3}-C\left(\varepsilon_{4}\right) N_{4}-C\left(\varepsilon_{8}\right) N_{8}\right) \int_{0}^{1} q^{2} d x \\
& -\left(\frac{k}{2} N_{8}-C\left(\varepsilon_{1}\right) N_{1}-\frac{k^{2}}{b} N_{2}-\varepsilon_{3} N_{3}-l k N_{5}-\left(k+\frac{1}{2}\right) N_{7}\right) \int_{0}^{1}\left(\varphi_{x}+\psi+l \omega\right)^{2} d x \\
& -\left(m N_{6}-N_{1} \varepsilon_{1}-2 l k_{0} \mu^{2} N_{5}-\frac{\mu^{2}}{2} N_{7}-C \mu N_{8}\right) \int_{0}^{1} z^{2}(x, 1, t) d x \\
& -N_{6} m \tau_{0} \int_{0}^{1} \int_{0}^{1} z^{2}(x, \rho, t) d \rho d x
\end{aligned}
$$

Then, we let

the choices yield

$$
N_{6}=1, N_{1}=N_{7}=l N_{5}=\frac{3}{\rho_{1}},
$$

$$
\begin{aligned}
\mathcal{L}^{\prime}(t) \leq & -\left(\frac{1}{2}-N \mu\right) \int_{0}^{1} \varphi_{t}^{2} d x-\left(\frac{3}{2}-N_{8} \varepsilon_{8}\right) \int_{0}^{1} \omega_{t}^{2} d x \\
& -\left(\frac{b}{2} N_{2}-\left(C+\frac{2 b^{2} l^{2}}{k}\right) \frac{3}{\rho_{1}}-\varepsilon_{3} N_{3}\right) \int_{0}^{1} \psi_{x}^{2} d x \\
& -\left(\frac{\rho_{2}}{2} N_{3}-\rho_{2} N_{2}-\varepsilon_{4} N_{4}-3-C\left(\varepsilon_{8}\right) N_{8}\right) \int_{0}^{1} \psi_{t}^{2} d x-\left(\frac{3 k_{0}}{2 \rho_{1}}-\varepsilon_{8} N_{8}\right) \int_{0}^{1}\left(\omega_{x}-l \varphi\right)^{2} d x \\
& -\left(\frac{\rho_{3}}{2} N_{4}-C N_{2}-C\left(\varepsilon_{3}\right) N_{3}-C\left(\varepsilon_{8}\right) N_{8}\right) \int_{0}^{1} \theta^{2} d x-m \tau_{0} \int_{0}^{1} z^{2}(x, \rho, t) d \rho d x \\
& -\left(\frac{k}{2} N_{8}-\left(C\left(\varepsilon_{1}\right)+\left(k+\frac{1}{2}\right)+k\right) \frac{3}{\rho_{1}}-\frac{k^{2}}{b} N_{2}-\varepsilon_{3} N_{3}\right) \int_{0}^{1}\left(\varphi_{x}+\psi+l \omega\right)^{2} d x \\
& -\left(N \beta-C N_{3}-C\left(\varepsilon_{4}\right) N_{4}-C\left(\varepsilon_{8}\right) N_{8}\right) \int_{0}^{1} q^{2} d x \\
& -\left(m-\left(\varepsilon_{1}+2 k_{0} \mu^{2}+\frac{1}{2} \mu^{2}\right) \frac{3}{\rho_{1}}-C \mu N_{8}\right) \int_{0}^{1} z^{2}(x, 1, t) d x .
\end{aligned}
$$


As follows, we need to choose our constants carefully. we let $\varepsilon_{1}=\frac{\rho_{1}}{6 m}$ and choose $N_{2}$ large, such that

$$
\frac{b}{2} N_{2}-\left(C+\frac{2 b^{2} l^{2}}{k}\right) \frac{3}{\rho_{1}} \geq \alpha_{1}>0 .
$$

At this point, we take $N_{8}$ large enough so that

$$
\frac{k}{2} N_{8}-\left(C\left(\varepsilon_{1}\right)+\left(k+\frac{1}{2}\right)+k\right) \frac{3}{\rho_{1}}-\frac{k^{2}}{b} N_{2} \geq \alpha_{2}>0,
$$

and then select $\varepsilon_{8}$ such that $\varepsilon_{8} \leq \min \left\{\frac{3}{2 N_{8}}, \frac{3 k_{0}}{2 \rho_{1} N_{8}}\right\}$. We choose $N_{3}$ large enough so that

$$
\frac{\rho_{2}}{2} N_{3}-\rho_{2} N_{2}-\varepsilon_{4} N_{4}-3-C\left(\varepsilon_{8}\right) N_{8} \geq \alpha_{3}>0,
$$

and choose $\varepsilon_{3}$ such that $\alpha_{1}-\varepsilon_{3} N_{3}>0$ and $\alpha_{2}-\varepsilon_{3} N_{3}>0$. We then choose $N_{4}$ large enough so that

$$
\frac{\rho_{3}}{2} N_{4}-C N_{2}-C\left(\varepsilon_{3}\right) N_{3}-C\left(\varepsilon_{8}\right) N_{8}>0
$$

and choose $\varepsilon_{4}$ such that $\alpha_{3}-\varepsilon_{4} N_{4}>0$. We set $N$ so large to satisfies

$$
N \beta-C N_{3}-C\left(\varepsilon_{4}\right) N_{4}-C\left(\varepsilon_{8}\right) N_{8}>0 .
$$

Finally, by taking $|\mu|$ so small that

$$
\frac{m}{2}-\left(2 k_{0} \mu^{2}+\frac{1}{2} \mu^{2}\right) \frac{3}{\rho_{1}}-C \mu N_{8}>0 .
$$

Utilizing the definition of $E(t)$, we have

$$
\mathcal{L}^{\prime}(t) \leq-c_{1} E(t) .
$$

On the other hand, exploiting (3.14), we get

$$
\left(N-c_{2}\right) E(t) \leq \mathcal{L}(t) \leq\left(N+c_{2}\right) E(t),
$$

which deduces that

$$
\mathcal{L}^{\prime}(t) \leq-k_{2} \mathcal{L}(t), \forall t>0 .
$$

A simple integration over $(0,1)$ leads to

$$
\mathcal{L}(t) \leq \mathcal{L}(0) e^{-k_{2} t} .
$$

It gives the desired result in Theorem 3.1 when combined with the equivalence of $\mathcal{L}(t)$ and $E(t)$.

Acknowledgment. This work was supported by the National Natural Science Foundation of China [grant number 11771216], the Natural Science Foundation of Jiangsu Province [grant number BK20151523], the Six Talent Peaks Project in Jiangsu Province [grant number 2015-XCL-020] and the Qing Lan Project of Jiangsu Province.

\section{References}

[1] T.A. Apalara, Well-posedness and exponential stability for a linear damped Timoshenko system with second sound and internal distributed delay, Electron. J. Differential Equations 2014 (254), 1-15, 2014.

[2] T.A. Apalara and S.A. Messaoudi, An exponential stability result of a Timoshenko system with thermoelasticity with second sound and in the presence of delay, Appl. Math. Optim. 71 (3), 449-472, 2015.

[3] M. Bresse, Cours de Mecanique Appliquee par M. Bresse rsistance des matriaux et stabilit des constructions, Mallet-Bachelier, Paris, 1859.

[4] A.C. Casal and J.I. Díaz, On the complex Ginzburg-Landau equation with a delayed feedback, Math. Models Methods Appl. Sci. 16 (1), 1-17, 2006. 
[5] M.M. Cavalcanti et al., Uniform decay rates for the energy of Timoshenko system with the arbitrary speeds of propagation and localized nonlinear damping, Z. Angew. Math. Phys. 65 (6), 1189-1206, 2014.

[6] M.M. Chen, W.J. Liu and W.C. Zhou, Existence and general stabilization of the Timoshenko system of thermo-viscoelasticity of type III with frictional damping and delay terms, Adv. Nonlinear Anal. 7 (4), 547-569, 2018.

[7] Z.J.Chen, W.J. Liu and D.Q. Chen, General decay rates for a laminated beam with memory, Taiwanese J. Math. 23 (5), 1227-1252, 2019.

[8] L.H. Fatori and J.E.M. Rivera, Rates of decay to weak thermoelastic Bresse system, IMA J. Appl. Math. 75 (6), 881-904, 2010.

[9] M. Kafini et al., Well-posedness and stability results in a Timoshenko-type system of thermoelasticity of type III with delay, Z. Angew. Math. Phys. 66 (4), 1499-1517, 2015.

[10] A.A. Keddi, T.A. Apalara and S.A. Messaoudi, Exponential and polynomial decay in a thermoelastic-Bresse system with second sound, Appl. Math. Optim. 77 (2), 315-341, 2018.

[11] V. Komornik, Exact controllability and stabilization, The multiplier method. MassonJohn Wiley, Paris, 1994.

[12] J.E. Lagnese, G. Leugering and E.J.P.G. Schmidt, Modelling of dynamic networks of thin thermoelastic beams, Math. Methods Appl. Sci. 16 (5),327-358, 1993.

[13] J.E. Lagnese, G. Leugering and E.J.P.G. Schmidt, Modeling, analysis and control of dynamic elastic Multi-Link structures, Systems \& Control: Foundations \& Applications, Boston, MA, 1994.

[14] G. Li, X.Y. Kong and W.J. Liu, General decay for a laminated beam with structural damping and memory: the case of non-equal wave speeds, J. Integral Equations Appl. 30 (1), 95-116, 2018.

[15] G. Li, Y. Luan, J.Y. Yu and F.D. Jiang, Well-posedness and exponential stability of a flexible structure with second sound and time delay, Appl. Anal. DOI: 10.1080/00036811.2018.1478081.

[16] G. Li, D.H. Wang and B.Q. Zhu, Well-posedness and decay of solutions for a transmission problem with history and delay, Electron. J. Differential Equations, 2016 (23), 1-21, 2016.

[17] G.W. Liu, Well-posedness and exponential decay of solutions for a transmission problem with distributed delay, Electron. J. Differential Equations, 2017 (174), 1-13, 2017.

[18] W.J. Liu and M.M. Chen, Well-posedness and exponential decay for a porous thermoelastic system with second sound and a time-varying delay term in the internal feedback, Contin. Mech. Thermodyn. 29 (3), 731-746, 2017.

[19] W.J. Liu, K.W. Chen and J. Yu, Asymptotic stability for a non-autonomous full von Kármán beam with thermo-viscoelastic damping, Appl. Anal. 97 (3), 400-414, 2018.

[20] W.J. Liu, D.H. Wang and D.Q. Chen, General decay of solution for a transmission problem in infinite memory-type thermoelasticity with second sound, J. Therm. Stresses 41 (6), 758-775, 2018.

[21] W.J. Liu, J.Y. Yu and G. Li, Exponential stability of a flexible structure with second sound, Ann. Polon. Math. DOI: 10.4064/ap171116-31-8.

[22] W.J. Liu and W.F. Zhao, Stabilization of a thermoelastic laminated beam with past history, Appl. Math. Optim. 80, 103-133, 2019.

[23] S. Nicaise and C. Pignotti, Stability and instability results of the wave equation with a delay term in the boundary or internal feedbacks, SIAM J. Control Optim. 45 (5), 1561-1585, 2006.

[24] S. Nicaise, C. Pignotti and J. Valein, Exponential stability of the wave equation with boundary time-varying delay, Discrete Contin. Dyn. Syst. Ser. S, 4 (3), 693-722, 2011. 
[25] S. Nicaise, J. Valein and E. Fridman, Stability of the heat and of the wave equations with boundary time-varying delays, Discrete Contin. Dyn. Syst. Ser. S, 2 (3), 559-581, 2009.

[26] A. Pazy, Semigroups of linear operators and applications to partial differential equations. Springer, New York, 1983.

[27] Y. Qin, J. Ren and T. Wei, Global existence, asymptotic stability, and uniform attractors for non-autonomous thermoelastic systems with constant time delay, J. Math. Phys. 53 (6), 063701, 1-20, 2012.

[28] M.L. Santos, D.S. Almeida Júnior and J.E. Muñoz Rivera, The stability number of the Timoshenko system with second sound, J. Differential Equations, 253 (9), 2715-2733, 2012.

[29] D.H. Wang, G. Li and B.Q. Zhu, Exponential energy decay of solution for a transmission problem with viscoelastic term and delay, Mathematics, 4 (42), 1-13, 2016.

[30] D.H. Wang, G. Li and B.Q. Zhu, Well-posedness and general decay of solution for a transmission problem with viscoelastic term and delay, J. Nonlinear Sci. Appl. 9 (3), 1202-1215, 2016.

[31] B. Wu, S.Y. Wu, J. Yu and Z.W. Wang, Determining the memory kernel from a fixed point measurement data for a parabolic equation with memory effect, Comput. Appl. Math. 37 (2), 1877-1893, 2018.

[32] S.T. Wu, Asymptotic behavior for a viscoelastic wave equation with a delay term, Taiwanese J. Math. 17 (3), 765-784, 2013.

[33] X.B. Zhang and H.L. Zhu, Hopf bifurcation and chaos of a delayed finance system, Complexity, Article ID: 6715036, 1-18, 2019.

[34] X.B. Zhang, H.Y. Zhao and Z.S. Feng, Spatio-temporal complexity of a delayed diffusive model for plant invasion, Comput. Math. Appl. 76 (11-12), 2575-2612, 2018. 